\title{
EFFECT OF RADIOTHERAPY ON RENAL FUNCTION IN CERVICAL CANCER PATIENTS TREATED AT A COMPREHENSIVE CANCER CENTRE IN NIGERIA
}

\author{
*1Otene S. A. \& ${ }^{2}$ Usman M. A. \\ ${ }^{*}$ Department of Oncology and Palliative, Care Federal Medical Centre Makurdi, Benue State, Nigeria \\ ${ }^{2}$ Department of Radiotherapy and Oncology, Usman Danfodiyo University Teaching Hospital Sokoto, Sokoto State, Nigeria \\ Corresponding Author E-mail: sammy4ng@yahoo.com, samuelotene@gmail.com Phone: +2348054456699
}

\begin{abstract}
Cervical cancer (CC) has been identified as a leading cause of cancer-related death of women in Nigeria. Unfortunately, treatment for CC induces renal function injury due to nephrotoxicity of commonly used cytotoxic medications and radiotherapy. The aim of this study was to determine whether radiotherapy consistently impairs the renal function of cervical cancer patients after treatment as claimed by literature. The study was an ex post facto research, for retrospective evaluation of documented information on 220 cervical cancer patients treated in the Department of Radiotherapy, Usman Danfodiyo University Teaching Hospital (UDUTH), Sokoto, Nigeria. The study covered a sixyear period from January, 2010 to December, 2015. Data were obtained from the patients' case notes using a semi-structured data extraction form. Independent t-test and chi-square statistics were used to obtain and interpret results from the analysis of the data. The study hypothesis one states that there would be both positive and negative effects of radiotherapy on the renal function of patients with cervical cancer. But no significant difference was found regarding the effect of radiotherapy on renal function of the patients with cervical cancer at $\chi^{2}$ (1, $\mathrm{N}=220)=.284, \mathrm{P}=288$. Hypothesis two states that there was no statistically significant difference in the Glomerular Filtration Rate (GFR) of the patients, measured before and after treatment $(\mathrm{t}(219)=-0.66, \mathrm{P}>0.5)$. Extrapolating from the study results revealed that radiotherapy does not only cause renal function impairment, but also enhances renal function among patients with cervical cancer. The study however recommends that there should be a constant monitoring of the GFR for every patient with cervical cancer underdoing radiotherapy.
\end{abstract}

Keywords: Cervical Cancer, Chemotherapy, Radiotherapy, Renal Function

LICENSE: This work by Open Journals Nigeria is licensed and published under the Creative Commons Attribution License 4.0 International License, which permits unrestricted use, distribution, and reproduction in any medium, provided this article is duly cited.

COPYRIGHT: The Author(s) completely retain the copyright of this published article.

OPEN ACCESS: The Author(s) approves that this article remains permanently online in the open access (OA) mode.

QA: This Article is published in line with "COPE (Committee on Publication Ethics) and PIE (Publication Integrity \& Ethics)". 


\section{INTRODUCTION}

Cancer of the cervix (CC) is the second commonest cancer affecting women worldwide and about $86 \%$ of cervical cancer cases occur in developing countries (Janaki, Mukesh, Arul Ponni, \& Numala, 2010). According to statistical data, 8-30 new cases of CC occur in 100,000 females within a year, depending on the region and state (Goga-Mega, Tozija, \& Spasovski, 2015). Cervical cancer is recognized to be one of the leading causes of illness and death among women worldwide, especially in developing countries (Janaki, Mukesh, Arul Ponni, \& Numala, 2010) (including Nigeria) (Okadome et al., 2018). Unfortunately, most of the patients in Nigeria present at late stages and often have evidence of renal impairment at presentation (Okadome et al., 2018). The renal system is one of the first body systems to be directly affected by cancer of the cervix, especially in advanced cases (Abdus-Salam, Bojude, and Abdus-salam, 2009; Janaki, Mukesh, Arul Ponni, and Numala, 2010). Renal impairment has been previously seen in more than a quarter of patients presenting with cancer of the cervix prior to commencement of treatment (Abdus-Salam, Bojude, and Abdus-salam, 2009). These complications are often preventable or reversible with prompt diagnosis and treatment (Mutambara et al., 2012). Treatment of cervical cancer commonly requires the use of chemotherapy, which can be given at the same time with radiotherapy, which is commonly known as concurrent chemotherapy/radiotherapy (CRT), or alone as neo-adjuvant or adjuvant chemotherapy. The treatment is usually stratified by stage to include both Brachytherapy and External Beam Radiation Therapy (EBRT), which is done by the use of CT-based treatment planning and conformal blocking (Bruheim, Guren, \& Skovlund, 2010).

Regarding the effect and outcome of chemotherapy and radiation therapy on renal functioning in patients, it has been shown that these treatments have a mixed effect, based on the classification of the cancer stages. For example, Okadema et al. (2018) showed that radiotherapy and chemotherapy may have adverse effects on renal and urological function, including a $\geq 20 \%$ reduction in the estimated Glomerular Filtration Rate (eGFR) of a relatively high percentage of women treated with pelvic radiotherapy and/or chemotherapy. Varlotto et al. (2006) also found that radiotherapy is associated with a rise in renal dysfunction (Varlotto, 2006). Studies have also shown that the presence of renal impairment in patients with carcinoma of the cervix is a poor prognostic indicator (Chao, Leun, \& Grisby, 1998). Patients with evidence of renal impairment from CC are said to be at stage 3b, according to the widely used International Federation of Obstetrics and Gynaecology (FIGO) staging for cancer of the uterine cervix (Musa et al., 2016).

However, other reports based on comparison tests, have shown a statistically significant difference between GFR values at the beginning vs. three months after using the therapy, while there was no difference between GFR values at three months into treatment vs. six months into treatment (Goga-Mega, Tozija, \& Spasovski, 2015). Specifically for patients with cervical cancer, studies have shown a significant statistical difference between the average values of GFR after therapy. This led Horan, McArdle, Martin, Collins and Faul (2006) to assert that pelvic radiation does not induce any deterioration of renal function or degree of hydronephrosis. Rather, the kidney function was found to improve at three and 6 months in the majority of patients. 


\begin{abstract}
AIMS AND OBJECTIVES
The primary aim of the study was to evaluate the effect of radiotherapy on the GFR of cervical cancer patients that underwent this treatment procedure at the department of Radiotherapy and Oncology, Usman Danfodiyo University Teaching Hospital (UDUTH), Nigeria. The objectives were to:

1. determine the effect of radiotherapy on renal function in patients with cervical cancer after treatment.

2. ascertain the significance difference in renal function of cervical patients before and after treatment with radiotherapy.

3. establish whether the effect of radiotherapy on the renal function of cervical cancer patients could be related to the stage of the disease.
\end{abstract}

\title{
RESEARCH HYPOTHESES
}

$\mathrm{HA}_{1}$ : There would be a significant positive or negative effect of radiotherapy on renal function in patients with cervical cancer.

$\mathrm{HA}_{2}$ : There would be a statistically significant difference in renal function of patients with cervical cancer before and after radiotherapy.

$\mathrm{HA}_{3}$ : The effect of radiotherapy on the renal function of cervical cancer patients might be related to the stage of the cancer disease.

\section{METHOD}

\section{RESEARCH DESIGN}

The study adopted an ex post facto design for retrospective evaluation of cervical cancer patients that underwent radiotherapy treatment at the Department of Radio-Oncology, Usman Danfodiyo University Teaching Hospital (UDUTH), Nigeria. The study covered a 6-year period of treatment for patients with cervical cancer, starting from January 2010 and December 2015. In this context, the authors did not manipulate the independent variables (radiotherapy and chemotherapy) to observe their effect on the dependent variables (Renal function, eGFR).

\section{PARTICIPANTS}

Participants were cervical cancer patients that received radiotherapy treatment in the department of RadiotherapyOncology UDUTH, Nigeria. The sample comprised of 220 selected case notes of cervical cancer patients that were exposed to radiotherapy treatment. The patients' demographic characteristics include sex, age, marital status, and occupation.

\section{MATERIALS}

Data was collected from secondary sources, comprising patients' case notes obtained in the archives of the department of Radio-Oncology, UDUTH, Nigeria. The treatment information documented in patients' notes was obtained using a semi-structured data extraction form. For the radiotherapy procedure, patients were simulated in a computed tomography simulator with thermoplastic immobilization device and positioning devices such as knee-rests, to 
reproduce the same position during treatment. Three-dimensional conformal radiotherapy was planned using the Monaco treatment planning system (version 5.0). Radiation was delivered by linear accelerator with $6 \mathrm{MV}$ or $10 \mathrm{MV}$ photons. Four conformal fields were used in treating all carcinoma of cervix patients. Adequate coverage of target volumes and sparing of organs at risk was achieved. Treatment verification with Electronic Portal Imaging Devices (EPID) was done. The GFR values were calculated using Cocroft-Gault equation at first clinic visit, and repeated at first follow-up after treatment (radiotherapy).

\section{STATISTICAL ANALYSIS}

The data were analyzed using the Microsoft excel, then exported into SPSS version 20 (Chicago IL) for windows; for statistical analysis. The data were analyzed for frequency distribution, generated for all categorical variables. Mean and standard deviation were determined for quantitative variables. Comparison of the differences of the patients' renal function status before treatment and after treatment was determined through the use independent t-test.

\section{RESULTS}

The purpose of the study was to evaluate the effect of radiotherapy on the GFR of cervical cancer patients. The results obtained from the statistical analysis is tabulated and interpreted as follows:

Table 1: Demographic Distribution of Cervical Cancer Patients

\begin{tabular}{lll}
\hline Age (Year) & Frequency & Percent \\
\hline $20-29$ & 9 & 4.1 \\
$30-39$ & 27 & 12.3 \\
$40-49$ & 69 & 31.4 \\
$50-59$ & 53 & 24.1 \\
$60-69$ & 47 & 21.4 \\
$70-79$ & 11 & 5.0 \\
$>80$ & 4 & 1.8 \\
House wives & 135 & 61.4 \\
Civil servants & 35 & 15.9 \\
Trader (Business) & 31 & 14.1 \\
Farmers & 14 & 6.4 \\
Others & 5 & 2.3 \\
\hline Total & 220 & 100 \\
\hline
\end{tabular}

The demographic distribution of patients on table 1 shows that the peak age incidence occurred with age range of 4049 year (31.4\%). The mean age of the cancer patients was 49.9, $\mathrm{SD} \pm 11.9$, and the age range was 24-87 years. 134 $(61.4 \%)$ of the patients studied were housewives. 
Table 2: Stages of cervical cancer presented by the patients

\begin{tabular}{lll}
\hline Stage & Frequency & Percent \\
\hline $1 \mathrm{~b}$ & 11 & 5.0 \\
$2 \mathrm{a}$ & 33 & 15.0 \\
$2 \mathrm{~b}$ & 51 & 23.2 \\
$3 \mathrm{a}$ & 49 & 22.3 \\
$3 \mathrm{~b}$ & 46 & 20.9 \\
$4 \mathrm{a}$ & 19 & 8.6 \\
$4 \mathrm{~b}$ & 11 & 5.0 \\
\hline Total & 220 & 100 \\
\hline
\end{tabular}

Table 2 shows the classification of cancer stages. Stage $1 \mathrm{~b}$ accounted for 11(5 \%), 2a 33(15\%), 2b 51(23.2\%), 3a 49 $(22.3 \%), 3 b 46(20.9 \%), 4 a 19(8.6 \%)$, and $4 b$ accounted for $11(5 \%)$.

Table 3: Distribution of effect of radiotherapy on eGFR of patient with cervical cancer

\begin{tabular}{|c|c|c|c|c|c|c|}
\hline \multirow[b]{2}{*}{ Variables } & \multicolumn{4}{|c|}{ Change in eGFR } & \multirow{2}{*}{$\chi^{2}$} & \multirow{2}{*}{$P<0.05$} \\
\hline & \multicolumn{2}{|c|}{ (N) Negative (\%) } & \multicolumn{2}{|c|}{ (N) Positive (\%) } & & \\
\hline $\begin{array}{l}\text { Stages of C } \\
\text { G1b }\end{array}$ & 13 & 63.6 & 20 & 36.4 & .284 & .288 \\
\hline G2a & 12 & 39.4 & 17 & 60.6 & & \\
\hline G2b & 21 & 49.0 & 28 & 51.0 & & \\
\hline G3a & 20 & 42.9 & 26 & 57.1 & & \\
\hline G3b & 12 & 43.5 & 7 & 56.5 & & \\
\hline G4a & 8 & 63.2 & 3 & 36.8 & & \\
\hline G4b & 106 & 72.7 & 114 & 27.3 & & \\
\hline Total & 100 & 48.2 & 100 & 51.8 & & NS \\
\hline
\end{tabular}

Table 3 shows that there is both positive and negative effect of radiotherapy on renal function of patients with cervical cancer after treatment. Yet, in analyzing these effects by percentages based on patients' disease stages, G1b indicates $63.6 \%$ negative effect and $36.4 \%$ positive effective. G2a $39.4 \%$ negative effect and $60.6 \%$ positive effect, G2b $49.0 \%$ negative effect and $51.0 \%$ positive effect. G3a $42.9 \%$ negative effect and $57.1 \%$ positive effect, G3b $43.5 \%$ negative effect and $56.5 \%$ positive effect. G4a $63.2 \%$ negative effect and $36.8 \%$ positive, G4b $72.7 \%$ negative effect and $27.3 \%$ positive effect. Overall, the finding shows $51.8 \%$ positive effect and $48.2 \%$ negative effect on renal function of the 220 patients. This result indicates that radiotherapy has an effect on the renal function of patients with cervical cancer after treatment; but the effect was not found to be absolutely related to the stage or grade of the cancer presented by 
the patients. Also, the effect of radiotherapy on renal function of the patients with cervical cancer was not found to be of statistically significant difference, at $\chi^{2}(1, \mathrm{~N}=220)=.284, \mathrm{P}=288$.

Table 4: Summary of independent t-test of difference of GFR before and after treatment

\begin{tabular}{llllllll}
\hline Variables & Group & N & M & SD & t & df & P = 0.05 \\
\hline \multirow{2}{*}{ GFR: } & Before & 220 & 69.67 & 19.48 & & & \\
& & & & & -0.66 .2 & 219 & 0.505 \\
& After & 220 & 70.87 & 20.38 & & & \\
\hline
\end{tabular}

Table 5 shows there was a marginal difference in the mean score of patients GFR before and after radiotherapy intervention. The mean scores $(M=69.67, \mathrm{SD}=19.48, \mathrm{~N}=220)$ for $\mathrm{GFR}$ before treatment and the mean scores of $(\mathrm{M}=70.87, \mathrm{SD}=20.38, \mathrm{~N}=146)$ for GFR after treatment. However, there was no statistically significant difference in the patients GFR between before and after treatment as was $(\mathrm{t}(219)=-0.66, \mathrm{P}<0.5)$.

\section{DISCUSSION}

$\mathrm{CC}$ is the second most common malignancy in women and it is a leading cause of cancer-related death for women in Nigeria. Unfortunately, treatment for cervical cancer induces renal function injury due to the impact of high doses of ionizing radiation on the renal system in the course of radiotherapy. This study seeks to investigate the change in Glomerular Filtration Rate (GFR) among CC patients that have undergone radiotherapy in the department of RadioOncology UDUTH, Sokoto.

The mean age of the cancer patients in this study was $49.9 \pm 11.9$, with an age range of $24-87$ years. The peak incidence of (31.4\%) of the disease occurred in people age 40-49 years, just as it has been established in previous researches done in Nigeria (Muhammed, Ahmed, Oluwale, \& Avidime, 2016; Babarinsa, Akang, \& Adewole, 1998; Airede, \& Malami, 2005). The statistical analysis on table 2 shows the classification of cancer stages presented by patients. Stage 1 b 5\%, stage2a $15 \%$, and 2 b $23.2 \%$, stage 3 a $22.3 \%$, and 3 b $20.9 \%$, stage 4 a $8.6 \%$, and 4 b was $5 \%$ respectively. This shows that there was $80 \%$ incidence of advanced disease (Stage $2 \mathrm{~b}$ and above). This corresponds to other similar studies ( $\mathrm{Li}$ et al., 2018; Anorlu, Orakwue, Oyenein, \& Abudu, 2012) which describe the late presentation of cervical cancer cases in Nigeria, compared to high-income countries where early presentation of cases predominates the disease (Adejoke, Kulasingam, \& Vimig, 2012). This supports the importance of screening and early testing, which significantly reduces the incidence of cervical cancer and increases early detection of new cases, thereby improving prognosis for survival following treatment (Ali, Kuekerls, \& Wassie, 2021).

Concerning discussion of hypotheses developed for testing in the study; firstly, hypothesis one states that there would be a significant positive or negative effect of radiotherapy on renal function in patients with cervical cancer. The statistical finding on this hypothesis shows a bidirectional effect of radiotherapy on renal function of patiens with cervical cancer. The bidirectional effect outcome suggests that application of radiotherapy as an intervention procedure in the treatment of cervical cancer has both negative and positive effects. This was found from 
the results of the analysis which shows $51.8 \%$ positive effect of radiotherapy on renal function and $48.2 \%$ of its negative effect, in the 220 cervical cancer patients treated. These findings support the evidence from Okadema et al. (2018), who assert that radiotherapy and chemotherapy may have adverse effects on renal and urological function. Their claim is supported by many prospective studies, which indicate that major genitourinary complications (or a significant rise in renal dysfunction) are associated with postoperative radiotherapy (Okadema et al. (2018).

On the contrary, despite the availability of extant literature showing that radiotherapy has a negative effect on renal function of the cervical cancer patients, there is also empirical evidence from studies that show also the positive effect of radiotherapy on renal function of patients with cancer of the cervix, proven in this current study. For example, Horan and colleagues found that pelvic radiation does not induce any deterioration of renal function or degree of hydronephrosis (Horan et al., 2006). Another study similarly shows that kidney function was improved between the third and sixth months, in the majority of their patients (Goga-Mega et al., 2015). Other studies have shown that the dose (Gy) of radiation given and the Stage of the disease (especially among patients with stages III and IV cancer) are often responsible for the renal complication imposed by the use of radiotherapy in treatment of cervical cancer. For example, it was found that that patients with $\mathrm{KV}_{20 \mathrm{~Gy}}$ values $<10 \%$ retained significantly better renal function than did patients with $\mathrm{KV}_{20 \mathrm{~Gy}}$ values $>10 \%(\mathrm{P}=0.002)$ (Kunogi, Yamaguchi, Terao, \& Sasai, 2021). The incidence of complications may be reduced to some extent by careful dose planning and continuous observation of the patient during the irradiation (Varlotto et al., 2006; Kunogi et al., 2021).

Secondly, hypothesis two states that there would be a statistically significant difference in renal functions of patients with cervical cancer before and after radiotherapy. Reporting on the second hypothesis, the result shows a marginal difference in the mean score of patients' GFR before and after radiotherapy intervention. The mean scores were $(M=69.67, S D=19.48, N=220)$ for $G F R$ before treatment and the mean scores were $(M=70.87, S D=20.38$, $\mathrm{N}=146$ ) for GFR after treatment. This marginal difference observed in the mean scores of the patients' GFR before and after treatment gave strength to the results on hypothesis one, which shows a variance of $51.8 \%$ positive effect and $48.2 \%$ of negative effect in the 220 cervical cancer patients evaluated in the study. Despite the findings that radiotherapy has both negative and positive effect on renal function, there was no statistically significant difference in the patients GFR before and after treatment at $(\mathrm{t}(219)=-0.66, \mathrm{P}<0.5)$. Better still, there was also no statistically significant difference regarding the effect of radiotherapy on renal function of patients with cervical cancer at $\chi^{2}(1$, $\mathrm{N}=220)=.284, \mathrm{P}=288$. This actually have clearly supported findings from previous studies, which have reported a no statistically significant difference in the risk between patients treated with radical hysterectomy with postoperative radiotherapy and radiotherapy alone (Li et al., 2018). However, the latency period between radiotherapy and the manifestation of urological complications may be relatively long, as radiotherapy has both acute and chronic sideeffects (Li et al., 2018). Similarly, Goga-Cmega et al., (2016) findings based on comparisons test revealed a significantly statistical difference between GFR values at the beginning vs. three months after using the therapy, baseline of treatment vs. six months after therapy while there was no difference between GFR values three months vs. six months later. 
Thirdly, hypothesis three states that the effect of radiotherapy on renal function of cervical cancer patients might be related to the stage/grade of the cancer disease. Consequently, it is interesting to report that the finding on this hypothesis was relatively accepted. The finding in the study statistically indicated that the effect of radiotherapy on renal function is not primarily related to the stage of the cancer presented for treatment. This was observed from the percentage analysis done from grouping of the patients' cervical cancer disease by grade/stages. The negative effect of radiation therapy was found on G1b 63.6\%, G2a 39.4\%, G2b 49.0\%, G3a 42.9\%, G3b 43.5\%, G4a 63.2\% and G4b $72.7 \%$. The positive effect recorded was on G1b 36.4\%, G2a 60.6\%, G2b 51.0\%, G3a 57.1\%, G3b 56.5\%, G4a 36.8\%, G4b 27.3\%.

But by virtue of the variation of these percentage results, the negative effect of radiotherapy on renal function was obviously found to be predominant in patients presenting with advance stages of the cervical cancer. These findings have supported Li et al. (2018) findings that radiotherapy has related urological complications actually in patients with cervical cancer FIGO stage IB-IIIB treated with radical radiotherapy or concomitant radical hysterectomy. Li et al. (2018) further states that in patients with locally advanced cervical cancer, radiotherapy may produce relatively low and acceptable rates of toxicity. Based on this assertion from Li et al. (2018), the incidence of complications may be reduced to some extent by careful dose planning and continuous observation of the patient during the irradiation (Chapman et al., 2019; Goga- Mega et al., 2016).

\section{CONCLUSION}

Cervical cancer is a prevalent pathology in women and it remains a disease of great public health concern in most of developing world, Nigeria inclusive. Apparently and unfortunately, various known modalities for managing this disease have been associated with documented deleterious effects on the renal function of patients treated. However, although it is commonly known that radiotherapy can impair the renal function of patients treated for cervical cancer, this study has found that it can also induce a very positive effect of on patients' renal function. Despite the findings that radiotherapy has both negative and positive effect on renal function, there was no statistically significant difference in the patients' GFR before and after treatment. Perhaps, this is because the injurious effect of radiotherapy to renal function is relatively a factor of the grade or stage of the cancer presented by patients during treatment. Better still, incidence of complications may be reduced to a large extent by careful dose planning and constant monitoring of the patient during the irradiation. Consequently, it is necessary and important for the radiation oncologists to maintain the standard dose applications and procedures for treatment of cervical cancer patients, as well as to monitor patients closely so as to either prevent complications or identify them early for management. 


\section{REFERENCES}

Anorlu, R.I., Orakwue, C.O., Oyeneyin. L, and Abudu, O.O. (2004). Late presentation of patients with cervical cancer to a tertiary hospital in Lagos: what is responsible? Eur J Gynaecol Oncol.; 25(6):729-32. PMID: 15597852 .

Abdus-salam, A.A., Bojude, A.D., and Abdus-salam, R.A. (2009). Renal status of patients with cervical cancer prior to treatment commencement. Tropical Journal of Nephrology; Vol.4 No1, p 17-20. Retrieved from https://www.tjn.online.com

Adegoke, O., Kulasingam, S., and Virnig, B. (2012). Cervical cancer trends in the United States: a 35-year population-based analysis. J Womens Health (Larchmt); 21(10):1031-7. Doi: 10.1089/jwh.2011.3385.

Ali, F., Kuelker, R, and Wassie, B. (2012). Understanding Cervical Cancer in the context of Developing Centres. Ann Trop Med Public Health; 5: 3-15.

Airede, L.R., and Malami, S.A. (2005). A five-year review of female genital tract malignancies in Sokoto, Northwestern Nigeria. Mary Slessor J Med; 5:51-6.

Babarinsa, A., Akang, E.E, and Adewole, I.F. (1998). Pattern of gynecological malignancies at the Ibadan cancer registry (1976-1995). Nig Q J Hosp Med; 8:103-6.

Bruheim, K., Guren, M.G., and Skovlund, E., et al. (2010). Late side effects and quality of life after radiotherapy for rectal cancer. Int J Radiat Oncol Biol Phys; 76: 1005-1011.

Chao, K.S., Leung, W.M., and Grigsby, P.W. et al. (1998). The clinical implications of hydronephrosis and the level of ureteral obstruction in stage IIIB cervical cancer, Int J Radiat Oncol Biol Phys. Mar 15; 40(5): 10951100 .

Chapman, B.V., Swanick, C.W., Ning, M.S., Allen, P.K., Soliman, P.T., and Westin, S.N., et al. (2019). Adjuvant combined-modality therapy for stage IIIC endometrioid and non-endometrioid endometrial cancer. Gynecol Oncol, 154: 22-8.

Goga-Cmega, V., Tozija, L., and Spasovski, G. (2016). Influence of radiation therapy on glomerular filtration rate after treating pelvic malignancy. Mac Med Review; 70(2): 88-93, DOI: 10.1515/mmr-2016-0017

Horan, G., McArdle, O., Martin, J,, Collins, C.D., and Faul, C. (2006). Pelvic radiotherapy in patients with hydronephrosis in stage IIIB cancer of the cervix: renal effects and the optimal timing for urinary diversion? Gynecol Oncol, 101:441-4.

Janaki, M.G., Mukesh, S., Arul Ponni., and Nirmala., S. (2010). Aggressive approach in a case of cancer cervix with uremia. Indian J Palliat Care; dio: 10:4103/0973-1075.63136

Kunogi, H., Yamaguchi, N., Terao, Y., and Sasai, K. (2021). dosimetric predictors of nephrotoxicity in patients receiving extended-field radiation therapy for gynecological cancer. Radiat Oncol; 16-25

Li. F., Guo, H., Qiu, H., Liu, S., Wang, K., Yang, C., Tang, C., Zheng, Q, and Hou, Y. (2018) Urological complications after radical hysterectomy with postoperative radiotherapy and radiotherapy alone for cervical cancer. Medicine; 97:13. Retrieved from http://dx.doi.org/10.1097/MD.0000000000010173 
Mohammed, A., Ahmed, S.A., Oluwole, O.P., and Avidime, S. (2006). Malignant tumours of the female genital tract in Zaria, Nigeria. Ann Afr Med; 5:93-6.

Musa, J., Nankat, J., Achenbach, C., Shambe., I., Taiwo, B., Mandong, B., Daru, P., Murphy, R., \& Sagay A. (2016). Cervical cancer survival in a resource-limited setting-North Central Nigeria. Infectious Agents and Cancer dio: 11. 10.1186/s13027-016-0062-0

Mutambara, J., Mutandwa, P., Mahapa, M., Chirasha, V., Nkiwane, S., and Shangahaidonhi, T. (2017) Knowledge, attitudes and practices of cervical cancer screening among women who attend traditional churches in Zimbabwe. Journal of Cancer Research and Practice, 4(2): 53-8.

Okadome et al. (2018). Renal function and urological complications after radical hysterectomy with postoperative radiotherapy and platinum-based chemotherapy for cervical cancer. Japanese Journal of Clinical Oncology, 48(2) 115-123 doi: 10.1093/jjco/hyx160

Varlotto, J.M., Gerszten, K., Heron, D.E., Comerci. J., Gautam, S., Selvaraj, R, et al. (2006). The potential nephrotoxic effects of intensity modulated radiotherapy delivered to the para-aortic area of women with gynecologic malignancies preliminary results. Am J Clin Oncol.; 29:281-9.ss 